\title{
Differences in the association between structural factors and diabetes self-monitoring support
}

\author{
Michiel J. Storimans · Olaf H. Klungel • \\ Herre Talsma $\cdot$ Cornelis J. de Blaey
}

Received: 25 November 2006/Accepted: 25 March 2007/Published online: 12 June 2007

(C) Springer Science+Business Media B.V. 2007

\begin{abstract}
Objective To determine if the association between the level of community pharmacy diabetes services and six of its reported determinants is influenced by the definition of these services.

Method Cross-sectional survey among 97\% of all Dutch community pharmacies $(1,642)$ registered in 2004. Seven definitions of self-monitoring support (support to patients performing self-monitoring of blood glucose) were constructed: one based on the Dutch pharmacy practice guideline (containing five activities related to patient counselling, calibration and dispensing), one based on patient counselling activities only and five definitions based on each separate activity. Multivariable models of selfmonitoring support according to the different definitions were compared.

Main Outcome Measure Associations between determinants and the different definitions of self-monitoring support, expressed as odds ratio (OR) and $95 \%$ confidence
\end{abstract}

M. J. Storimans ( $\square)$ · O. H. Klungel · C. J. de Blaey Department of Pharmacoepidemiology and Pharmacotherapy, Utrecht Institute for Pharmaceutical Sciences, Utrecht University, Sorbonnelaan 26, Utrecht, The Netherlands e-mail: michiel.storimans@organon.com

M. J. Storimans · C. J. de Blaey

Scientific Institute Dutch Pharmacists, The Hague,

The Netherlands

H. Talsma

Department of Biopharmaceutics, Utrecht Institute for Pharmaceutical Sciences, Utrecht University, Utrecht,

The Netherlands intervals $(95 \% \mathrm{CI})$. All definitions were compared to the practice guideline definition.

Results The ORs of 14 of the 48 possible comparisons of different definitions were significantly different from one. The standardized difference ranged from 1.42 (95\% CI: $1.01-1.90)$ to 3.05 (95\% CI: $1.51-4.61)$. Three out of six predictive models retained different determinants compared to the multivariable model of self-monitoring support based on the guideline.

Conclusion The association between self-monitoring support and its determinants depend on the definition of self-monitoring support. This underlines the importance for pharmacy practice research to unambiguously describe the characteristics and the setting of an intervention. Only with a complete description of the intervention, the likelihood for success of implementation in another setting can be determined.

Keywords Community pharmacy services - Type 1 diabetes - Type 2 diabetes - Pharmacy practice research . Cross-sectional survey $\cdot$ Intervention - Self-monitoring of blood glucose

\section{Impact of findings on practice}

- The association between pharmaceutical care activities targeted on self-monitoring of blood glucose and its determinants is dependent on what exactly comprises the care activity.

- Interpretation differences may inhibit the practice implementation of the support of effective blood glucose self-monitoring on a wide scale. 


\section{Introduction}

Pharmaceutical care interventions have shown that community pharmacists can have a significant impact on patient outcomes [1-4]. International and national guidelines encourage community pharmacies to take active interest in care for diabetic patients [5-7].

What exactly comprises the community pharmacy services to diabetes patients is still widely discussed. Intervention studies aiming to improve diabetes care in community pharmacy all emphasize different activities. For example, Jaber et al. focused on improvement of diabetes care on patient counselling, while the Asheville project and Rothman et al. also included structured followup of patients in their diabetes care improvement program $[2,4,8]$. Pharmacy practice guidelines, although generally reviewing similar topics, vary on the level of detail of the recommendations [5-7]. Moreover, they only describe the best practice situation. In daily routine, the feasibility of these recommendations and the actual implementation is diverse [9, 10].

This diversity in the aspects that constitute diabetes care is a key issue, especially for implementation research. Studies into implementing pharmaceutical care report, among other things, physical lay-out of the pharmacy, computer support, knowledge and competence as well as lack of time and reimbursement as key implementation factors [11, 12]. Moreover, the impact of these structural factors for successful implementation may not be the same for different aspects of (diabetes) care. For example, improving the pharmacy team's knowledge is likely to be an important aspect when implementing educational programs on life style changes. It is probably less relevant for areas in which, traditionally, pharmacies have more expertise, such as improving patient compliance.

The objective of this study was to determine if the association between structural pharmacy-related factors and the provision of support to patients performing selfmonitoring of blood glucose (self-monitoring support) changes when different definitions of self-monitoring support are used.

\section{Methods}

Setting and study design

We gathered data using a cross-sectional survey among all Dutch community pharmacies registered in January 2004 ( $n=1,691)$. Data acquisition comprised two stages. First, semi-structured interviews were held with seven pharmacists to identify relevant structural factors potentially associated with self-monitoring support. These seven pharmacists also pre-tested the final survey and found it to be comprehensive. A pilot study was performed among 50 randomly selected community pharmacies to determine feasibility as well as a limited validation on variability in responses and comprehensibility. If an item was checked by less than $10 \%$ of the respondents, the item or the response categories were rephrased or deleted. The results of these questionnaires were excluded from the final analysis.

In the second stage, a mailing was sent in February 2004 to all Dutch community pharmacies not involved in the pilot study. It was addressed to the senior pharmacist in the pharmacy. The invitational letter stated that the internetbased survey (respondents could fill in the questionnaire by accessing an exclusive internet site) was intended for the pharmacist responsible for the (diabetes) care activities. Participants could respond anonymously. After three weeks, all pharmacies received a reminder and a paper version of the survey, which could be returned at no cost.

Of all respondents, sites which functioned as an annex of another pharmacy (limited services available and only open a few hours per day) were excluded. In most regions only a few pharmacists dispense outside of office hours. This 'out of hours' service rotates among all pharmacies in that region. However, in some regions, a specialized 'out of hours' pharmacy has been created, which conducts all 'out of hours' dispensing. These pharmacies were excluded from the analysis as well.

\section{Definition of self-monitoring support}

Seven different definitions of self-monitoring support were constructed (see Table 1). (Insert Table 1, Description of the different definitions of blood glucose self-monitoring support here). All definitions were based on the Dutch pharmacy practice guideline [5]. This guideline defines five separate support activities in three areas of pharmacy services to patients performing self-monitoring. These areas were: patient counselling, calibration of testing equipment, and providing blood glucose meters at no costs to patients performing SMBG for a short period. Patients in the Netherlands who use oral hypoglycemic agents usually do not receive (full) reimbursement of the blood glucose meter; therefore some pharmacists accommodate patients with one, to reduce initial costs of self-monitoring. Counselling was subdivided into three domains: choice of a suitable blood glucose meter, the operation of that blood glucose meter and performing the test procedure. Respondents were asked which support activities they performed. All responses were dichotomous (yes or no). Services that were not prompted by the pharmacy, for example patient counselling after a specific question from the patient, were excluded. 
Table 1 Description of the different definitions of blood glucose self-monitoring support based on the Dutch pharmaceutical care guideline for type 2 diabetes mellitus ${ }^{\mathrm{a}}$ [5]

\section{Description}

Definition The community pharmacy supports patients with the

1 calibration of their blood glucose meters $(45.8,270)$

Definition The community pharmacy provides patient counselling on

2 the type of blood glucose meter most suitable for them $(41.9,247)$

Definition The community pharmacy provides patient with an 3 instruction on the operation of their blood glucose meter $(69.5,410)$

Definition The community pharmacy provides patient counselling $4 \quad$ on the procedure of blood glucose testing $(64.7,382)$

Definition The community pharmacy temporarily accommodates

5 patients with a blood glucose meter at no cost (34.9, 206)

Definition The community pharmacy provides patient counselling on 6 the use of the blood glucose meter and testing procedure $(65.1,384)$

Definition The community pharmacy performs at least three of the 7 activities mentioned in definitions 1 to $5(55.3,326)$

${ }^{a}$ Between brackets the number of respondents who reported to provide self-monitoring support according to the definition \% ( $n$ )

Definitions 1 to 5 represented the five separate aspects of self-monitoring support. For definition 6, we only included the aspects on patient counselling activities. As reference we used the practice guideline definition, comprising all five aspects of self-monitoring support (definition 7).

\section{Pharmacy-related determinants}

Apart from data on the pharmacy self-monitoring support, the 47-item questionnaire also gathered information on five structural factors that were mentioned in the first stage of the study as being essential to the implementation: availability of separate counselling area, having a pharmacy technician specialized in diabetes, perceived knowledge of the pharmacy's team on self-monitoring of blood glucose, perceived workload, and lastly existence of a regional agreement on the division of roles in self-monitoring support. These structural factors were reported as relevant in previous studies into barriers for implementation of pharmaceutical care and diabetes care [11-16]. Moreover, the factors represent key issues in many intervention programs intended for improving diabetes care in community pharmacies (training, specialization, improving collaboration).

Perceived knowledge of the pharmacy team on selfmonitoring was collected with three statements concerning the ability of the pharmacy staff to provide self-monitoring support. Workload was collected as agreement to a statement on high workload being a reason not to provide support to all patients. All four items were scored on a four-point scale (entirely disagree to entirely agree). Because respondents might not always be the actual proprietor of the pharmacy, we only included respondents who agreed to a statement that they could independently determine the level of services in self-monitoring support.

Analysis

The results of the three questions regarding a team's knowledge showed significant correlation. Therefore, we calculated the sum score of all questions regarding knowledge, ranging from zero to nine. The sum score was categorized into three groups, representing the tertiles. The first tertile (a sum score of five or less) was used as reference.

For definitions 6 and 7, the total number of activities was calculated. Since no consensus on the relative importance of these activities exists, all activities were weighed equally. For the practice guideline definition, we classified a pharmacy as a low level service pharmacy (LLS pharmacies; performing two or less of the five separate aspects) or a high level service pharmacy (HLS pharmacies; providing three, four or all of the aspects). Definition 6 was categorized as high level counselling services (counselling on type of blood glucose meter and counselling on operation of blood glucose meter) or low level counselling services (no counselling on type of blood glucose meter or no counselling on operation of blood glucose meter).

To study the effect of different definitions on the associations with the structural factors, we first calculated odds ratios (ORs) for the association between the five structural factors and provision of self-monitoring support according to the different definitions using logistic regression. These ORs were compared both visually and statistically. However, since all ORs were derived from the same dataset, comparison using confidence intervals $(95 \% \mathrm{CI})$ of the ORs was not possible. We therefore calculated a standardized odds ratio by dividing the OR for the association between a structural factor and the provision of self-monitoring support, according to definitions 1 to 6 , by the OR for the association between that structural factor and providing self-monitoring support based on definition 7. A mean standardized OR was determined for 2,000 replications of the dataset using a bootstrapping method. The $95 \%$ CI of this standardized odds ratio were derived from the distribution of these replications. The mean standardized OR was calculated for every structural factor separately. Using this method we compared definitions 1 to 6 with the definition based on the practice guideline (definition 7).

The univariable models were extended to multivariable predictive models. We used backwards elimination based on changes in log likelihood ratio with a cut-off value of 0.10 and compared which structural factors remained 
statistically significantly associated with the provision of self-monitoring support.

\section{Results}

Of all 1,642 pharmacies in the main study, 757 returned the questionnaire. After exclusion of annexes and 'out of hours' pharmacies, $724(44 \%)$ remained. A total of 134 respondents did not agree with the statement that they could decide on the pharmacy's policy with diabetes care independent of the actual proprietors of the pharmacy.

Among the remaining 590 pharmacies, $65 \%$ of the respondents offered patient counselling on the testing procedure, $70 \%$ offered instruction on the operation of the blood glucose meter and $42 \%$ provided counselling on the choice of blood glucose meter. Calibration of blood glucose meters was reported by $46 \%$ of the respondents and providing a patient with a meter at no cost by $35 \%$ of the respondents. Using definition 7 (practice guideline), 45\% pharmacies were classified as LLS pharmacies and $55 \%$ as high level service pharmacies.

Figure 1 reports the odds ratios of the univariable associations between the different definitions of self-monitoring support and the five structural factors. (insert Fig. 1 Crude association between structural factors and selfmonitoring support according to different definitions here). Apart from the factor 'separate counselling area' and 'perceived workload, low level service pharmacies differ significantly from HSL pharmacies for all definitions used in this study. Moreover, the associations are robust, indicated by the fact that for every structural factor, they all point in the same direction.

Table 2 shows the results of the comparison of different definitions of pharmacy services for the five structural factors. A standardized odds ratio that is significantly different from 1 illustrates that the association between a structural factor and the level of services as defined by that definition is not similar to the association between the same structural and the level of services as defined by the reference definition. As shown by the fact that most standardized odds ratio are larger than 1, the association between self-monitoring support as described in definition 7 and the structural factors is often stronger than the association between the separate aspects of self-monitoring support and the structural factor. In 14 out of the 48 comparisons, these differences were statistically significant. Especially for 'knowledge', 'specialized diabetes technician' and 'local collaboration', the association with support as described in definition 7 is different compared to the association with its separate aspects. Interestingly, the strength of the association between the structural factors also varied amongst the separate aspects (definitions 1 to 5). Nevertheless, the differences were less pronounced (data not shown).

Limiting the definition of self-monitoring support only to patient counselling activities (definition 6), resulted in different associations with three out of the five structural factors observed compared to definition 7. Only the association with 'separate counselling area' and 'perceived workload' was not significantly different between these two definitions.

As can be seen in Table 3 , the results of the multivariable model using different aspects of self-monitoring support led to dissimilar models. (insert Table 3 Factors associated in a multivariable model using different definitions of self-monitoring support here). Only 'knowledge' was statistically significant in all models and 'workload' did not remain associated in any model. Compared to the multivariable model with self-monitoring support defined
Fig. 1 Crude association between structural factors and self-monitoring support according to different definitions (odds ratio and 95\% confidence intervals. (a) Separate counselling area, (b) Specialised diabetes technician, (c) Local collaboration, (d) Pharmacy's team knowledge of self-monitoring of blood glucose, (e) Perceived workload
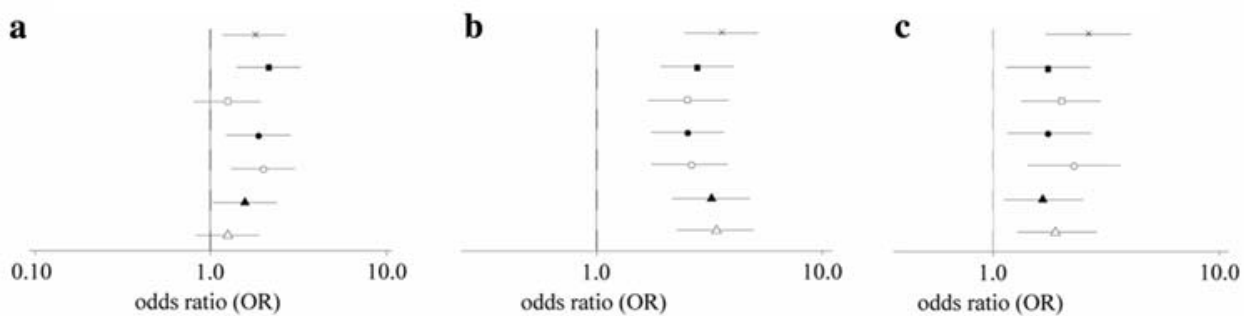

d

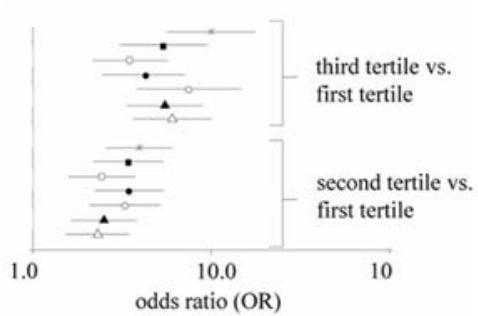

e

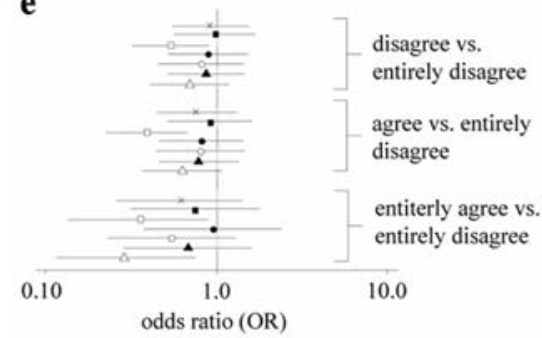

$\times$ definition 7

- definition 6

definition 5

- definition 4

definition 3

4 definition 2

$\Delta$ definition 1 
Table 2 Ratio of the association between different structural factors and self-monitoring support according to definitions 1-6 and the association between different structural factors and self-monitoring support according to definition $7^{\mathrm{a}}$

\begin{tabular}{|c|c|c|c|c|c|c|}
\hline & Def. 1 & Def. 2 & Def. 3 & Def. 4 & Def. 5 & Def. 6 \\
\hline \multicolumn{7}{|c|}{ Separate counselling area } \\
\hline available & $1.45(0.96-2.05)$ & $1.15(0.82-1.53)$ & $0.90(0.66-1.19)$ & $0.97(0.72-1.29)$ & $1.45(0.94-2.10)$ & $0.83(0.64-1.06)$ \\
\hline not available & reference & reference & reference & reference & reference & reference \\
\hline \multicolumn{7}{|c|}{ Specialised diabetes technician } \\
\hline available & $1.10(0.74-1.63)$ & $1.13(0.81-1.46)$ & $1.42(1.01-1.90)$ & $1.44(1.05-1.95)$ & $1.44(0.91-2.10)$ & $1.31(1.00-1.73)$ \\
\hline not available & reference & reference & reference & reference & reference & reference \\
\hline \multicolumn{7}{|c|}{ Local collaboration on self-monitoring support } \\
\hline available & $1.43(0.91-2.11)$ & $1.60(1.14-2.18)$ & $1.17(0.80-1.66)$ & $1.51(1.08-2.03)$ & $1.34(0.84-1.92)$ & $1.51(1.15-2.06)$ \\
\hline not available & reference & reference & reference & reference & reference & reference \\
\hline \multicolumn{7}{|c|}{ Knowledge of self-monitoring ${ }^{b}$} \\
\hline first tertile & reference & reference & reference & reference & reference & reference \\
\hline second tertile & $1.77(1.16-2.67)$ & $1.61(1.14-2.27)$ & $1.22(0.84-1.72)$ & $1.15(0.79-1.60)$ & $1.67(1.04-2.66)$ & $1.16(0.82-1.53)$ \\
\hline third tertile & $1.74(0.84-3.05)$ & $1.87(1.13-2.92)$ & $1.35(0.78-2.14)$ & $2.43(1.55-3.58)$ & $3.05(1.51-4.61)$ & $1.86(1.24-2.65)$ \\
\hline \multicolumn{7}{|c|}{ Perceived workload is a reason for not giving self-monitoring support to all patients } \\
\hline entirely agree & $2.46(0.84-5.41)$ & $0.98(0.41-2.16)$ & $1.17(0.50-1.93)$ & $0.67(0.27-1.18)$ & $1.94(0.79-4.52)$ & $0.82(0.41-1.38)$ \\
\hline agree & $1.28(0.72-2.12)$ & $0.99(0.64-1.42)$ & $0.98(0.62-1.56)$ & $0.97(0.63-1.52)$ & $2.02(1.12-3.40)$ & $0.86(0.58-1.23)$ \\
\hline disagree & $1.38(0.80-2.15)$ & $1.06(0.70-1.54)$ & $1.16(0.73-1.75)$ & $1.05(0.68-1.59)$ & $1.77(0.98-2.79)$ & $0.95(0.63-1.33)$ \\
\hline entirely disagree & reference & reference & reference & reference & reference & reference \\
\hline
\end{tabular}

${ }^{a}$ Bootstrap-method with 2,000 replications, mean (95\% Bca confidence limits)

${ }^{\mathrm{b}}$ First tertile (sum score: 0-5; lowest perceived knowledge); second tertile (sum score: 6); third tertile (sum score: 7-9; highest perceived knowledge)

Table 3 Factors associated in a multivariable model using different definitions of self-monitoring support ${ }^{\mathrm{a}}$

\begin{tabular}{|c|c|c|c|c|c|}
\hline & $\begin{array}{l}\text { Specialised } \\
\text { diabetes technician }^{\mathrm{b}}\end{array}$ & $\begin{array}{l}\text { Separate counselling } \\
\operatorname{area}^{\mathrm{b}}\end{array}$ & Workload $^{\mathrm{b}}$ & $\begin{array}{l}\text { Local } \\
\text { collaboration }^{\mathrm{b}}\end{array}$ & $\begin{array}{l}\text { Knowledge of } \\
\text { pharmacy team }^{\text {b }}\end{array}$ \\
\hline Definition 1 & $2.72(1.74-4.23)$ & & & & $\begin{array}{l}2.07(1.33-3.20) \\
4.47(2.63-7.60)\end{array}$ \\
\hline Definition 2 & $2.51(1.60-3.94)$ & & & & $\begin{array}{l}2.20(1.41-3.42) \\
4.12(2.45-6.92)\end{array}$ \\
\hline Definition 3 & & $1.69(1.06-2.70)$ & & $1.93(1.19-3.13)$ & $\begin{array}{l}2.95(1.83-4.75) \\
6.16(3.10-12.2)\end{array}$ \\
\hline Definition 4 & $1.90(1.24-2.92)$ & & & $1.46(0.94-2.28)$ & $\begin{array}{l}2.98(1.88-4.74) \\
3.35(1.89-5.96)\end{array}$ \\
\hline Definition 5 & $1.73(1.10-2.73)$ & & & $1.66(1.10-2.49)$ & $\begin{array}{l}2.28(1.46-3.58) \\
2.43(1.45-4.05)\end{array}$ \\
\hline Definition 6 & $1.92(1.25-2.96)$ & $1.71(1.08-2.72)$ & & & $\begin{array}{l}2.86(1.79-4.55) \\
4.66(2.52-8.60)\end{array}$ \\
\hline Definition 7 & $2.40(1.55-3.73)$ & & & $2.11(1.34-3.32)$ & $\begin{array}{l}3.54(2.25-5.57) \\
6.49(1.45-11.9)\end{array}$ \\
\hline
\end{tabular}

a Backwards elimination, $n=478$.

b Association only presented for factor that significantly improved multivariable model $(P$-value of log likelihood ratio $>0.1)$

according to definition 7, only self-monitoring support defined as providing patients with a blood glucose meter at no cost and counselling on testing procedure were similar. In these three models, 'specialized diabetes technician', 'local collaboration' and 'knowledge' remained statisti- cally significantly associated with the outcome. Models of the other aspects did not include 'local collaboration' or did include 'separate counselling area'. The aspects pertaining information on counselling activities all resulted in different multivariable models. Moreover, when we only 
included patient counselling activities in our definition, we observed a multivariable model that was different from definition 7 of self-monitoring support as well as from the models with self-monitoring support defined according to the separate aspects.

Correlation among the different aspects was relatively high. The minimal Spearman $R^{2}$ was 0.178 for the bivariate correlation between lending of blood glucose meter and counselling on the type of blood glucose meter. The highest $R^{2}$ was 0.667 for the correlation between counselling on the testing procedure and instruction on the operation of blood glucose meters. All correlations were statistically significantly different from 1. Correlations between the separate aspects on self-monitoring support and the composite score on self-monitoring support ranged from 0.402 to 0.677 (all $P<0.01$ ).

\section{Discussion}

This is one of the first studies to assess the consequence of the fact that pharmaceutical care is not a single activity, but comprises many different aspects in pharmacy practice research. We found that associations between structural factors and separate aspects of self-monitoring support were often different from associations between structural factors and composite scores of self-monitoring support.

Variation in the associations with structural factors was not only observed for the five separate support activities defined in the practice guideline. Also when we compared two different ways to sum self-monitoring support (definition 6 and definition 7), the associations with the most structural factors were statistically different.

These differences in the strength of the associations were observed in the univariable models. Furthermore, multivariable analysis resulted in dissimilar models. However, probably as a result of the strong correlation between the structural factors, the effect of different definitions of self-monitoring support was most pronounced for the univariable associations.

With respect to our findings, improvement of selfmonitoring support is best achieved through investing in pharmacy's team knowledge of self-monitoring. Since our results imply that this structural factor is associated with all separate aspects of self-monitoring support as well as with the definition based on the practice guideline, it is the most effective precondition to change. On the other hand, perceived workload was not associated with any aspect of selfmonitoring support, indicating that it is not relevant for the provision of community pharmacy support to diabetes patients. However, due to the cross-sectional nature of this study, a longitudinal study is required to verify this.
We developed our own questionnaire, since to our knowledge, no validated questionnaire on structural factors in diabetes care or pharmacy's self-monitoring support exists. Because we had no gold standard to compare our results, we focused on the face-validity and content validity. The pilot study showed sufficient response and contrast on our main determinants and outcome variables.

\section{Limitation}

Response rates for this type of study are generally low, as it was with our questionnaire. Non-response can introduce a significant bias if for example pharmacists with no interest in pharmaceutical care decline to participate. However, had this been true, we would have expected a relatively high proportion of respondents who had participated in the nation-wide diabetes care improvement project. Yet, $48 \%$ of the pharmacists had participated in this nation-wide project, which is a similar response rate compared to the nation-wide participation rate of this improvement project (45\%) [17]. Furthermore, all results were self-reported which may have introduced a reporting information bias. We tried to minimize this effect by guarantying anonymity of the respondents.

We used a definition based on the current Dutch pharmaceutical care standard to provide a reference for our objective: what is the relevance of the definition of the care process when studying the relationship between structural components and the process? However, to fully appreciate the potential impact of pharmacy services on self-monitoring, more research is needed comprising not only data on care activities but also on patient outcomes.

Implications

We used self-monitoring support as an example of pharmaceutical care to diabetes patients, since it was considered a care activity that could be made operational without much risk for information bias. It is a relatively homogenous topic that has distinct aspects recognizable to all respondents. It is somewhat difficult to infer to what extent our conclusions apply to other fields of pharmaceutical care. Defining care on a higher hierarchical level would result in including more diverse activities. In that case, observed differences in associations between different aspects of care and structural factors would probably also become larger.

As our results show, the different activities comprising self-monitoring support cannot be compared with respect to the different structural factors associated with them. This implies that structural factors are of limited use when determining the quality of 'self-monitoring support' by 
community pharmacies. The opposite is also true. When measuring processes in a community pharmacy, it is imprudent to use a process that in fact comprises a plethora of activities. This also affects studies that use self-monitoring support as outcome variable, for example practice research of implementation strategies. Without proper and unequivocal definitions of the observed process, incorrect conclusions on facilitators and barriers of diabetes care in community pharmacies could be drawn.

\section{Conclusion}

The association between structural factors and selfmonitoring support varies when different definitions of self-monitoring support are used. However, this variation depends on which structural factor is studied. Our data do not answer the vital question of which definition is most appropriate for intervention and implementation studies. To clarify this, more research into the relation between the process of self-monitoring support and patient outcomes is needed. Still, we have shown that it is of pertinent importance that studies on implementation of diabetes care activities and diabetes care intervention research explicitly state which aspects comprise their definition of diabetes care.

Funding This study was made possible by an unrestricted grant from the Royal Dutch Society for the Advancement of Pharmacy (KNMP), The Hague, The Netherlands.

\section{References}

1. Coast-Senior E, Kroner B, Kelley C, Trili L. Management of patients with type 2 diabetes by pharmacists in primary care clinics. Ann Pharmacother 1998;32:636-641.

2. Rothman R, Malone R, Bryant B, Horlen C, Pignone M. Pharmacist-led, primary care-based disease management improves hemoglobin A1c in high-risk patients with diabetes. Am J Med Qual 2003;18:51-8.
3. Cranor CW, Christensen DB. The Asheville Project: short-term outcomes of a community pharmacy diabetes care program. J Am Pharm Assoc (Wash) 2003;43:149-59.

4. Cranor CW, Bunting BA, Christensen DB. The Asheville Project: long-term clinical and economic outcomes of a community pharmacy diabetes care program. J Am Pharm Assoc (Wash) 2003;43:173-84.

5. WINAp. FPZ-standaard Diabetes mellitus type 2 [Pharmaceutical care standard diabetes mellitus type 2]. The Hague, The Netherlands: WINAp; 2000.

6. Royal Pharmaceutical Society of Great Britain. Practice guidance for community pharmacists on the care of people with diabetes. London: Royal Pharmaceutical Society of Great Britain; 2001.

7. Europharmforum. Improved quality in diabetes care: the pharmacist in St Vincent team: protocol and guidelines. [mongraph on the Internet] 2000 [cited on 2004 Nov 25]. Available from: http:www.euro.who.int/document/e75680.pdf.

8. Jaber LA, Halapy H, Fenret M, Tummalapalli S, Diwakaran H. Evaluation of a pharmaceutical care model on diabetes management. Ann Pharmacother 1996;30:238-243.

9. Stewart D, O' Shea J, McCaig D. Agreement of practitioners with the recommendations of the 'guidelines for community pharmacists on the care of patients with diabetes'. J Soc Adm Pharm 2003;20:13-17.

10. Younis WS, Campbell S, Slack MK. Pharmacists' attitude toward diabetes and their involvement in diabetes education. Ann Pharmacother 2001;35:841-845.

11. Bell HM, McElnay JC, Hughes CM. A qualitative investigation of the attitudes and opinions of community pharmacists to pharmaceutical care. J Soc Adm Pharm 1998;15:284-295.

12. Odedina FT, Segal R, Hepler CD. Providing pharmaceutical care in community practice: differences between providers and nonproviders of pharmaceutical care. J Soc Adm Pharm. 1995;12:170-180.

13. van Mil J, de Boer W, Tromp T. European barriers to the implementation of pharmaceutical care. Int $\mathrm{J}$ Pharm Pract 2001;9:163-168.

14. Hughes C, McCann S. Perceived interprofessional barriers between community pharmacists and general practitioners: a qualitative assessment. Br J Gen Pract 2003;53:600-6.

15. Dijkstra R, Braspenning J, Uiters E, van Ballegooie E, Grol R. Perceived barriers to the implementation of diabetes guidelines in hospitals in The Netherlands. Neth J Med 2000;56:80-5.

16. Brown J, Harris S, Webster-Bogaert S, et al. The role of patient, physician and systemic factors in the management of type 2 diabetes mellitus. Fam Pract 2002;19:344-9.

17. Beijer H, Essink R, de Blaey CJ, et al. Meer contacten en betere zorg. [More contact and better care] Pharm Weekbl 2004;139:1069-71. 